\title{
Terminal Relaxation and Diffusion of Entangled Three-Arm Star Polymers: Temperature and Molecular Weight Dependencies
}

\author{
K. L. NGAI, C. M. ROLAND \\ Naval Research Laboratory, Washington, DC 20375-5320
}

Received 13 January 1997; revised 27 May 1997; accepted 2 June 1997

\begin{abstract}
Recent experimental investigation of the terminal relaxation in high molecular weight polyisoprenes by dynamic mechanical measurements (C. A. Bero and C. M. Roland, Macromolecules, 29, 1562 (1996)) has found the terminal relaxation times to be more sensitive to changes in temperature for three-arm stars than for the linear polyisoprenes. Moreover, these measurements, carried out with significantly higher molecular weight samples than heretofore, show that the molecular weight dependence of the terminal relaxation times for three-arm star polyisoprenes is much weaker than the exponential dependence previously proposed (L. J. Fetters, et al., Macromolecules, 26, 647 (1993)). Tracer diffusion of labeled linear and three-arm star polyethylenelike diffusant molecules in a highly entangled linear polyethylene matrix exhibit temperature and molecular weight dependencies similar to those observed spectroscopically from bulk polymers. Both the temperature and molecular weight dependencies for the star-branched polymers are at variance with the predictions of the reptation model. It is shown here, however, that these observations can be reconciled through application of the coupling model of relaxation. (c) 1997 John Wiley \& Sons, Inc. J Polym Sci B: Polym Phys 35: 2503-2510, 1997
\end{abstract}

Keywords: rheology; diffusion; star-branched polymers; polyisoprene; terminal viscosity; molecular weight dependence; temperature dependence

\section{INTRODUCTION}

Comparisons of the temperature dependencies of the zero shear viscosity and diffusion coefficient of both star-branched polymers and their linear counterparts have been used to check the consistency of theoretical models. ${ }^{1-11}$ Experimentally, it is found that branching usually confers greater sensitivity to temperature, examples including commercial polyethylene,,${ }^{7,12}$ polybutadiene, ${ }^{13}$ hydrogenated polybutadiene (HPB), ${ }^{7,12-14}$ polyisoprene ${ }^{15,16}$ and hydrogenated polyisoprene. ${ }^{13}$ Analysis of some of the data for HPB has shown consistency ${ }^{6,8,12}$ with the coupling model of relax-

Correspondence to: C. M. Roland

Journal of Polymer Science: Part B: Polymer Physics, Vol. 35, 2503-2510 (1997) (C) 1997 John Wiley \& Sons, Inc.

CCC 0887-6266/97/152503-08 ation $(\mathrm{CM}) \cdot{ }^{17,18}$ According to the $\mathrm{CM},{ }^{6-10,17-19} \mathrm{a}$ linear polymer, which may be considered a twoarm star, will necessarily experience weaker intermolecular coupling for its terminal motions than a multiarm star polymer of the same arm molecular weight. ${ }^{1}$ It is an extant prediction of the CM that more intermolecularly coupled motions will be more sensitive to temperature changes. Thus, the CM predicts that the temperature dependence of star-branched polymers will invariably be stronger than that of the corresponding linear polymer, provided there is no additional contributing factor. On the other hand, the HPB data are also consistent with the reptation model, ${ }^{1-5}$ which posits an arm retraction mechanism as enabling reptation of branched chains. ${ }^{1-3}$ As pointed out by Graessley, ${ }^{5}$ such retraction produces a more compact transient struc- 
ture, having a concentration of gauche conformers higher than at equilibrium. Since these gauche conformers often have a higher energy than the trans states, the activation energy barrier for the terminal relaxation time, $\tau_{\eta}$, will be increased. Under this circumstance, the expectation from reptation theory is that star polymers will have a stronger temperature dependence than their linear counterparts.

Polyisoprene (PI) is distinct from the other, previously investigated, polymers, in that its conformers have virtually the same energy. ${ }^{5,20}$ Consequently, a change in population of the trans and gauche states, as putatively occasioned by arm retraction, would not alter the activation energy. Hence, the reptation prediction is that starbranched and linear polyisoprenes should have the same temperature dependence. ${ }^{5,13,21}$ In ref. 21 , it was asserted that star and linear PI have identical temperature dependence; however, no supporting data were given. Recently, terminal relaxations in linear and three-arm star polyisoprenes were reinvestigated using dynamic mechanical ${ }^{15}$ and dielectric normal mode measurements. ${ }^{16}$ The terminal relaxation times, as determined by either spectroscopy, were found to be more sensitive to changes in temperature for the stars than for the linear polyisoprene. This observed trend is at variance with the reptation model with or without the additional modification proposed by Graessley. ${ }^{5}$ However, it is consistent with the expectation from the CM. Moreover, the magnitude of the excess temperature dependence of star polyisoprene was consistent with the greater breadth of its terminal relaxation functions.

The higher molecular weight star studied by Bero and Roland ${ }^{15}$ has a significantly higher molecular weight than those measured previously by Fetters et al. ${ }^{21}$ From this earlier study, ${ }^{21}$ it was determined that the terminal viscosities for threeand four-arm polyisoprenes could be described by (in units of $\mathrm{Pa} \mathrm{s}$ )

$$
\eta=(4.47)\left(\frac{M_{a}}{M_{e}}\right)^{3 / 2} \exp \left(0.47 \frac{M_{a}}{M_{e}}\right)
$$

where $M_{a}$ is the arm molecular weight and $M_{e}$ is the entanglement molecular weight equal to 6400 $\mathrm{g} / \mathrm{mol}^{22,23}$ for PI. The exponential dependence of $\eta$ on $M_{a}$ was predicted by the reptation model. ${ }^{1-3}$ The data in ref. 15 cast doubt on the validity of this dependence for PI. As seen in Figure 1, the higher molecular weight star has a viscosity that

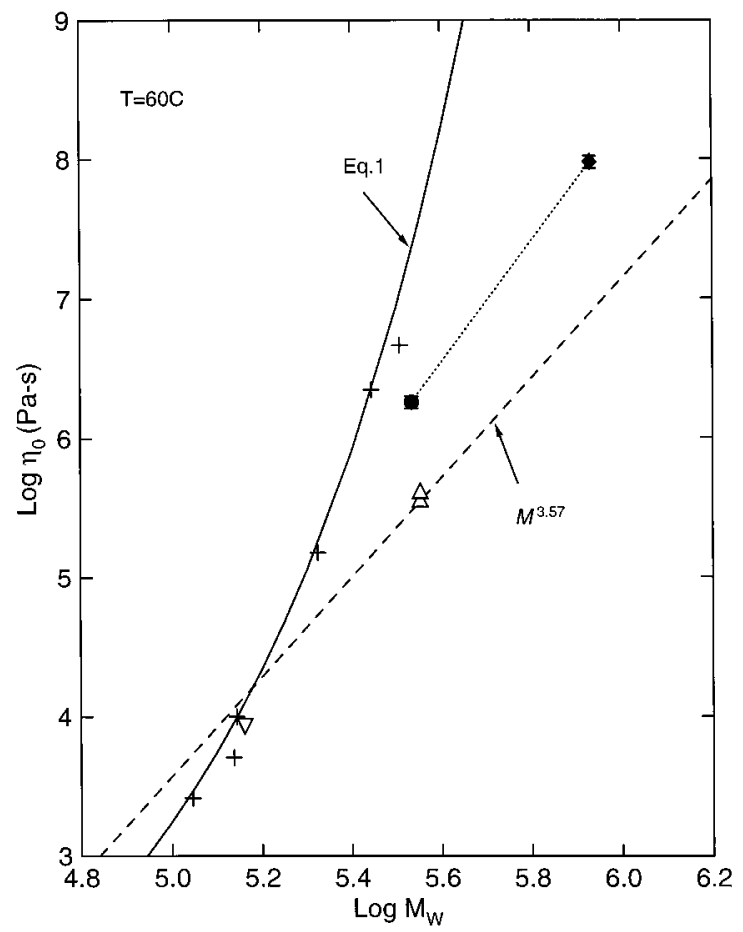

Figure 1. Zero-shear viscosities measured ${ }^{21}$ for the two 3-arm star PIs, S342 and S854 (filled symbols), and the linear PIs, L145 $(\nabla)$ and L357 $(\triangle)$. Errors of measurements are indicated. The dashed line is a fit to the data of linear PI obtained in refs. 19 and 20. The crosses $(+)$ are data for 3-arm star PIs, and the solid curve is the exponential molecular weight dependence (eq. 1), taken from ref. 19.

is more than 4 orders of magnitude smaller than the prediction from eq. 1 . While there is agreement for the shorter arm stars measured in ref. 21 , clearly the viscosity of star-branched PIs does not continue to increase exponentially when $M_{a} /$ $M_{e}$ is as high as 44 .

The coupling model offers simultaneous predictions (described in section II) of the molecular weight and temperature dependencies of the terminal relaxation time or viscosity. As presented in section III, the coupling parameter determined from the temperature dependence of the star PI's provides a prediction for the dependence of $\eta$ on $M_{a}$ that is consistent with the experimental data of ref. 15.

Finally, in section IV we revisit tracer diffusion data for linear and three-arm-star 1,4-polybutadiene in a highly entangled linear polyethylene matrix, as measured by Klein and co-workers ${ }^{24}$ many years ago. The experimental data indicate that the activation enthalpy (molecular weight dependence) of diffusion is significantly larger for 
the stars than for the linear polymer. Analysis of the molecular weight and temperature dependencies of the tracer diffusion coefficient is carried out, leading to conclusions similar to those deduced from the PI viscosity data.

\section{THEORETICAL BACKGROUND}

Several years ago, attempts were made to apply the coupling model (CM) to viscous flow and diffusion of entangled melts and concentrated solutions. ${ }^{6-10,19}$ These early applications of the model to linear polymer systems were overly simplified, based on the slowing down of isotropic Rouse modes without consideration of the lateral (i.e., tubelike) nature of the dynamic constraints imposed by other chains. The CM makes predictions concerning the shape of the terminal dispersion, as well as the molecular weight, $M$, and temperature, $T$, dependencies of the terminal relaxation time or, equivalently, the zero shear viscosity, $\eta$. The original version of the CM is not consistent with all experimental data, in particular failing to accurately describe the shape of the terminal dispersion in monodisperse linear polymers, as measured mechanically or dielectrically. ${ }^{19,26}$ An initial effort to address this shortcoming has been proposed, ${ }^{27}$ which attempts to take into consideration the specific nature of the entanglement constraints, as well as their subsequent mitigation.

The time scale, $\tau_{\mathrm{LCM}}$, for lateral constraint mitigation (LCM) is of the order of the terminal relaxation time. The coupling parameter is expected to have a constant value, $n_{\eta}$, at times shorter than $\tau_{\mathrm{LCM}}$, but decreases as $t$ approaches and exceeds $\tau_{\mathrm{LCM}}$. The decrease in coupling strength would be due to mitigation of the topological constraints supplied by other chains, as a result of their motions over the time scale of the terminal relaxation. This resulting modification of the $\mathrm{CM}^{27}$ causes the calculated time (or frequency) dependence of the terminal relaxation to become considerably narrower than that given by the correlation function,

$$
C(t)=\exp -(t / \tau)^{1-n_{\eta}}
$$

originally suggested by the CM. ${ }^{6-10}$ At least for dielectric spectroscopy, there is reasonable agreement with experimental data (i.e., the normal mode dispersion) of monodisperse, type A poly- mers. ${ }^{26}$ In any case, the original predictions of the CM for the $T$ - and $M$-dependences of $\tau_{\eta}$ or $\eta,{ }^{6-11}$

$$
\eta(M, T)=\tau_{\eta} / J_{e}^{0} \propto M^{2\left(1-n_{\eta}\right)}\left(\zeta_{0}(T)\right)^{1 /\left(1-n_{\eta}\right)}
$$

and the center of mass diffusion correlation time, ${ }^{7,8} \tau_{D}$,

$$
\tau_{D} \propto M^{2 /\left(1-n_{D}\right)}\left(\zeta_{0}(T)\right)^{1 /\left(1-n_{D}\right)}
$$

and the diffusion coefficient, $D$,

$$
D(M, T)=R_{g}^{2} / \tau_{D} \propto M^{1-2 /\left(1-n_{D}\right)}\left(\zeta_{0}(T)\right)^{-1 /\left(1-n_{D}\right)}
$$

remain valid, since this are governed by the initial (maximum) value of the coupling parameter (i.e., before constraint mitigation commences). In these equations, $J_{e}^{0}$ is the steady-state recoverable shear compliance, $\zeta_{0}(T)$ is the friction factor before entanglement coupling is taken into account, and $n_{\eta}$ and $n_{D}$ are the (constant) values of the coupling parameters for $t \ll \tau_{\text {LCM }}$ for respectively viscous deformation and diffusion. The characteristic time $\tau_{D}$ is the time for a mean square displacement of the molecular center of mass equal to the square of the radius of gyration, $R_{g}^{2}$. The coupling parameters $n_{\eta}$ and $n_{D}$ differ because viscous deformation and center of mass selfdiffusion are not exactly the same mode of motion, the former related to a two-point correlation function, while the center of mass $\left(R_{c m}\right)$ diffusion, described by $\left\langle R_{c m}(t) R_{c m}(0)\right\rangle$, involves a one point correlation function. Different $n_{\eta}$ and $n_{D}$ in eqs. 3 and 4 have accounted for the mismatch of the molecular weight dependence ( $M^{3,4}$ for $\tau_{\eta}$ and $M^{3.0}$ for $\tau_{D}$ ) found in many linear polymers, and for the differences in activation enthalpy, $E_{\eta}=7.2$ $\mathrm{kcal} / \mathrm{mol}$ for viscous deformation ${ }^{6-9,14}$ and $E_{D}$ $=6.2 \mathrm{kcal} / \mathrm{mol}$ for linear hydrogenated polybutadiene ${ }^{6-9,24,25,28}$ and $E_{\eta}=6.35 \mathrm{kcal} / \mathrm{mol}$ for viscous deformation $^{6-9,29}$ and $E_{D}=5.47 \mathrm{kcal} / \mathrm{mol}^{6-9,25,29}$ for linear polyethylene.

For multistar polymers, the more severe entanglement constraints are expected to increase the coupling parameter..$^{11}$ Thus, we expect (using the subscripts $L$ and $S$ to refer to linear and star polymers, respectively)

$$
n_{\eta S}>n_{\eta L}
$$

and 


$$
n_{D S}>n_{D L}
$$

when the molecular weight per arm, $M_{a}$, of the star substantially exceeds the entanglement molecular weight, $M_{e}$. However, when $M_{a} / M_{e}$ is not large, as is the case in many experimental investigations, $n_{\eta S}$ and $n_{D S}$ may be an increasing function of $M_{a} / M_{e}$, with inequalities 5 and 6 not yet holding. Equations 2 and 3 are valid for both linear and star-branched polymers; ${ }^{6-11}$ consequently, the $M$ - and $T$-dependences of the viscosities of the linear and star polymers, denoted respectively by $\eta_{L}$ and $\eta_{S}$, are related to each other by

$$
\eta_{S}(M, T) \propto\left(\eta_{L}(M, T)\right)^{1-n_{\eta L} / 1-n_{\eta S}}
$$

There is a corresponding expression,

$$
\tau_{D S}(M, T) \propto\left(\tau_{D L}(M, T)\right)^{1-n_{D L} / 1-n_{D S}}
$$

relating the $M$ - and $T$-dependences of the diffusion correlation times of star and linear polymers, $\tau_{D S}$ and $\tau_{D L}$. When inequalities 5 and 6 hold, the exponents on the right-hand side of eqs. 7 and 8 are larger than unity and consequently $\eta_{S}\left(\tau_{D S}\right.$ or $D_{S}$ ) of a star polymer has a stronger temperature and molecular weight dependence than $\eta_{L}\left(\tau_{D L}\right.$ or $D_{L}$ ) of the linear counterpart.

When the temperature dependence of the transport coefficients is Arrhenius (i.e. $\eta_{S} \propto \exp \left(E_{\eta S} /\right.$ $R T), \eta_{L} \propto \exp \left(E_{\eta L} / R T\right), \tau_{D S} \propto D_{S}^{-1} \propto \exp \left(E_{D S} / R T\right)$ and $\left.\tau_{D L} \propto D_{L}^{-1} \propto \exp \left(E_{D L} / R T\right)\right)$, the relations 6 and 7 between the $T$-dependences reduce to

$$
E_{\eta S}=E_{\eta L}\left(1-n_{\eta L}\right) /\left(1-n_{\eta S}\right)
$$

and

$$
E_{D S}=E_{D L}\left(1-n_{D L}\right) /\left(1-n_{D S}\right)
$$

This relation between the activation enthalpies $E_{\eta S}$ and $E_{\eta L}$ has been observed in terminal relaxation data of linear and star-branched hydrogenated polybutadiene. ${ }^{6-9,14}$

\section{ANALYSIS OF LINEAR AND THREE-ARM STAR POLYISOPRENE MECHANICAL DATA}

The zero-shear viscosities of the linear polyisoprene (PI) determined as a function of the weight

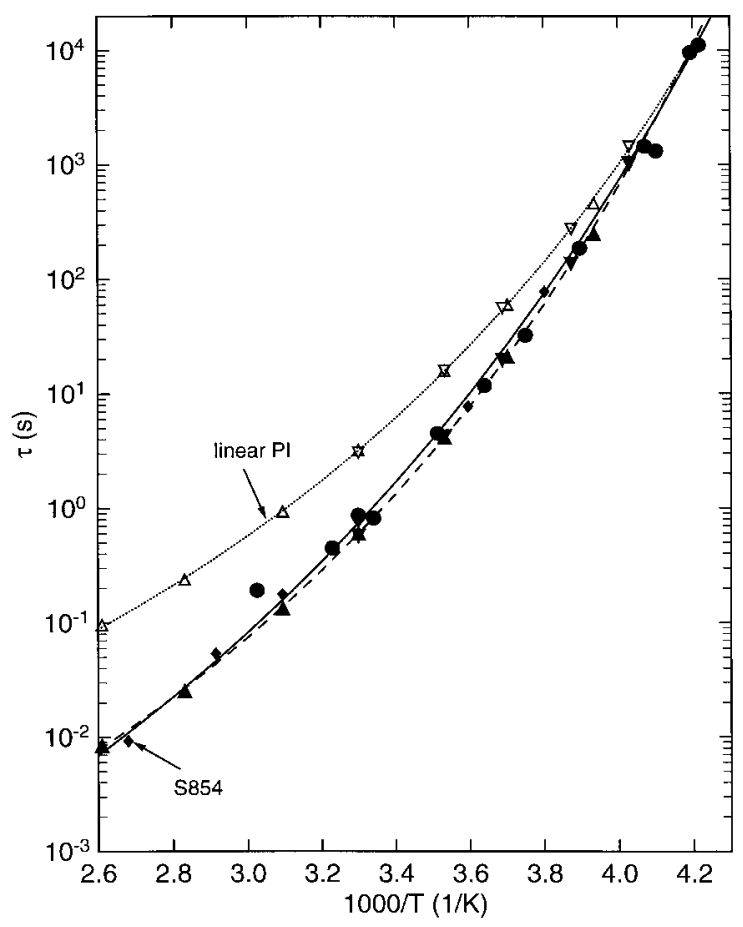

Figure 2. Terminal relaxation times for the linear PI (L145, inverted open triangles, and L357, open triangles), with the values for the lower molecular weight polymer shifted vertically by a factor of 24 . Terminal relaxation times for the two star-branched PI's (S854, filled diamonds, and S342, filled circles ) with the values of the smaller star shifted vertically upward by a factor of 6.4 (filled circles ). The dotted and solid curves represent VFTH eqs. 11 and 12, respectively. The dashed curve (filled triangles) represents the prediction from eq. 11 (open triangles) by using eqs. 10, 14, and 15 .

averaged molecular weight, $M_{w}$, in refs. 15 and 21 can be described by

$$
\eta_{L}=5.26 \times 10^{-15} M_{w}^{3.57}
$$

in units of $\mathrm{Pa} \mathrm{s}$ at $60^{\circ} \mathrm{C}$ (Fig. 1). The molecular weight dependence of $\eta_{L}$ is in good agreement with earlier measurements by Nemoto et al. ${ }^{30}$ and with dielectric normal mode relaxation times for PI. ${ }^{16,26}$ In a comparison of the experimental $M_{w}$-dependence (eq. 9) with the CM prediction (eq. 3), the coupling parameter of linear PI is deduced to have the value of

$$
n_{\eta L}=0.44
$$

In Figure 2 are shown the terminal relaxation times, $\tau_{\max L}$, defined as the reciprocal of the angular frequency of the maximum in the loss modu- 
Table I. Sample Designations

\begin{tabular}{llc}
\hline Designation & \multicolumn{1}{c}{ Polymer } & $M_{w}$ \\
\hline S342 & 3-arm star polyisoprene & 342,000 \\
S854 & 3-arm star polyisoprene & 854,000 \\
HDPE1 & polyethylene & high polymer \\
DJK5 & deuterated polybutadiene & 28,000 \\
DJK6 & deuterated polybutadiene & 38,000 \\
DWG & deuterated polybutadiene & 70,000 \\
\hline
\end{tabular}

lus, $G^{\prime \prime}(\omega)$, of the linear PI's with $M_{w}=3.57 \times 10^{5}$ (open triangles) and $1.45 \times 10^{5}$ (open inverted triangles ), respectively. All data for the lower molecular weight PI have been shifted vertically by a factor of 24 to superpose the results for the two linear polymers. The dotted curve represents a fit to the temperature dependence of $\tau_{\max L}$ by the Vogel-Fulcher-Tamman-Hesse (VFTH) equation $^{31-33}$

$$
\tau_{\max L}=1.47 \times 10^{-4} \exp \left(\frac{1453.1}{T-157.56}\right)
$$

Also shown in Figure 2 are the measured terminal relaxation times, $\tau_{\max S}$, of the star-branched polyisoprene samples S854 (filled diamonds) and S342 (filled circles) (see Table I). The data for S342 have been shifted vertically by a factor of 6.4 to superpose with the data for the higher molecular weight star S854. The superpositioning is adequate, although the two stars do not have identical temperature dependencies. ${ }^{15}$ The solid curve in Figure 2 represents the VFTH equation

$$
\tau_{\max S}=7.03 \times 10^{-7} \exp \left(\frac{2222.9}{T-142.82}\right)
$$

Since the relaxation times, $\tau_{\max L}$ and $\tau_{\max S}$, have the same temperature dependencies as the viscosities, $\eta_{L}$ and $\eta_{S}$, respectively, the temperature dependence in the proportionality relation 7 is equivalent to

$$
\tau_{\max S}(T) \propto\left(\tau_{\max L}(T)\right)^{1-n_{\eta L} / 1-n_{\eta S}}
$$

By choosing a reference temperature, $T_{\text {ref }}$, we convert the proportional relation 13 to

$$
\frac{\tau_{\operatorname{maxS}}(T)}{\tau_{\operatorname{maxS}}\left(T_{\operatorname{ref}}\right)}=\left(\frac{\tau_{\max L}(T)}{\tau_{\max L}\left(T_{\text {ref }}\right)}\right)^{1-n_{\eta L} / 1-n_{\eta S}}
$$

With the coupling parameter $n_{\eta L}$ already determined from the molecular weight dependence of $\eta_{L}$ (eq. 10), and with the shift factors, $\tau_{\max L}(T) /$ $\tau_{\max L}\left(T_{\text {ref }}\right)$ and $\tau_{\operatorname{maxS}}(T) / \tau\left(T_{\text {ref }}\right)$, known from experiment, eq. 14 can be used to determine $n_{\eta S}$. The temperature dependence of $\tau_{\operatorname{maxS}}(T)$ is calculated from that of $\tau_{\max L}(T)$ using eqs. 10, 11, and 14, with the choice of

$$
n_{\eta S}=0.54 \pm 0.02
$$

As shown as the dashed curve and filled triangles in Figure 2, this agrees quite well with the experimental data ( solid curve, filled diamonds, and filled circles). Of course this value for $n_{\eta S}$ is an approximation for the two stars, S854 and S342, since they have neither exactly the same temperature dependence nor identical terminal dispersions. ${ }^{15}$

The coupling parameters for linear PI and its three-arm counterpart given by eqs. 10 and 15 clearly satisfy inequality 5 . The star PI having a larger coupling parameter than linear PI is consistent with the greater breadths of the terminal relaxation functions observed for the stars. ${ }^{20,21}$ Moreover, eq. 7 guarantees that $\eta_{S}$ has a stronger molecular weight dependence than $\eta_{L}$. We can now assess whether the observed molecular weight dependence (Fig. 1) is consistent with eq. 7 , using the coupling parameter values determined from the temperature dependence of the relaxation times. The prediction, that the viscosity of S854 will be a factor of $53 \pm 8$ larger than $\eta_{S}$ for S342, is in agreement with the experimental data of Figure 1 ( solid diamond for S854 and solid circle for S342).

This test for consistency of the CM with the observed molecular weight dependence can be contrasted with the exponential molecular weight dependence (eq. 1), obtained by fitting the viscosity data of stars with shorter arm lengths by Fetters et al. ${ }^{19}$ to the reptation model. The reptation 
prediction is a viscosity increase of almost 6 orders of magnitude in going from S342 to S854 rather than the observed change of merely a factor of roughly 50 .

\section{ANALYSIS OF TRACER DIFFUSION OF ENTANGLED THREE-ARM STAR DATA}

In the last section we demonstrated a mutual consistency between the molecular weight and the temperature dependencies of the viscoelastic terminal relaxation in linear polyisoprene and its star-branched counterpart. ${ }^{20,21}$ Once the coupling parameters, $n_{\eta L}$ and $n_{\eta S}$, have been fixed by comparing the predicted molecular weight dependence of $\eta_{L}$ and temperature dependence of $\eta_{S}$, respectively, with experimental data, eq. 7 offers effectively a parameterless test of the coupling model. A similar situation occurs in the tracer diffusion of linear monodispersed deuterated 1,4polybutadienes (DPD) and their three-arm star counterparts in highly entangled linear polyethylene (HDPE1) melts and in melts of hydrogenated polybutadiene. Such experiments have been reported by Klein, Fletcher, and Fetters (KFF) ${ }^{24}$

The tracer diffusion coefficient of linear DPD molecules in HDPE1, $D_{L}$, has the molecular weight and temperature dependencies ${ }^{24}$

$$
D_{L} \propto M^{-1.95} \exp \left(-\frac{E_{D L}}{R T}\right)
$$

with

$$
E_{D L}=6.2 \mathrm{kcal} / \mathrm{mol}
$$

From the relation between $D_{L}$ and the diffusion correlation time of the linear molecule, $\tau_{D L}$, given by eqs. $4 \mathrm{a}$ and $4 \mathrm{~b}$, we have

$$
\tau_{D L} \propto M_{S}^{2.95} \exp \left(\frac{E_{D L}}{R T}\right)
$$

In a comparison of the molecular weight dependence of $\tau_{D L}$ obtained experimentally and that predicted by eq. $4 \mathrm{a}$, the coupling parameter for diffusion of linear chains is deduced to be

$$
n_{D L}=0.32
$$

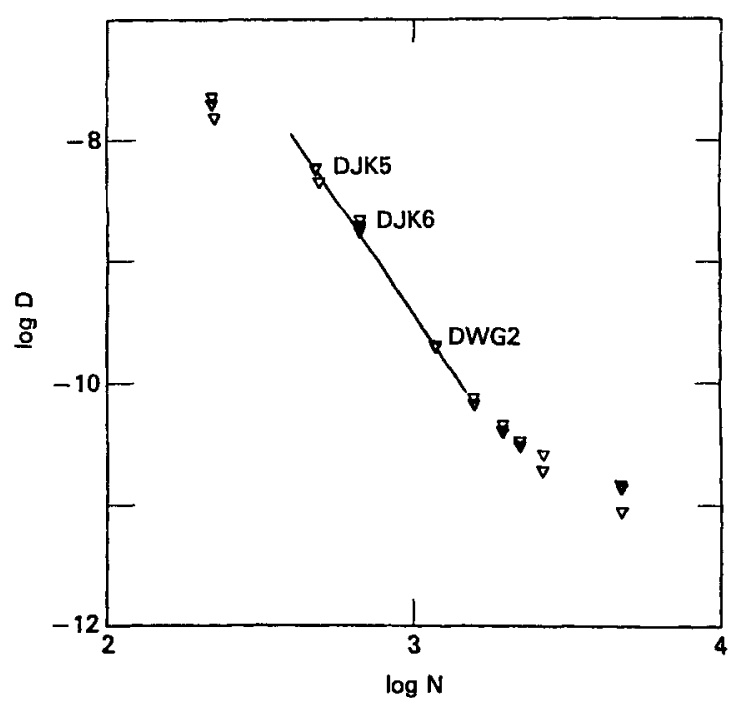

Figure 3. Experimental tracer diffusion data for starbranched DPD molecules in HDPE 1 at $176^{\circ} \mathrm{C}^{24}$ plotted against the degree of polymerization, $N$. The three starbranched molecules DJK5, DJK6, and DWG2 (see text) are indicated. The predicted approximate $M^{3.87}$ dependence of $D_{S}$ within the molecular weight range of these three stars is indicated by the straight line.

The tracer diffusion coefficient, $D_{S}$, of three-arm star DPD in HDPE1 measured at $176^{\circ} \mathrm{C}^{24}$ are shown in Figure 3. Herein we consider only data for the stars denoted by KFF as DJK5, DJK6, and DWG2 (and labeled as such in Fig. 3), which have total degrees of polymerization, $N$, equal to 490 , 660 , and 1200 , respectively ( see Table I). The reason for this restriction is that the activation enthalpies of diffusion of these three stars have been measured and they have nearly the same activation enthalpy, $E_{D S}$. Similar to the considerations for star-branched polyisoprenes in the previous section, an equivalent temperature dependence for the three PDP stars implies that their coupling parameters are the same. This equality makes the calculation of the variation of $D_{S}$ with molecular weight in the range defined by the three stars possible without introduction of additional parameters.

The value of the coupling parameter for the three stars can be determined from the experimental data. The $E_{D S}$ values are $11.2 \pm 2,9.1 \pm 0.7$, and $10.98 \pm 1.2 \mathrm{kcal} / \mathrm{mol}$ for DJK5, DJK6, and DWG2, respectively. ${ }^{24}$ Thus, within the experimental error, the three stars can be considered to have the same activation enthalpy, $E_{D S}=10.43$ $\mathrm{kcal} / \mathrm{mol}$, the mean value. The temperature dependence of $D_{S}$ for the three stars has approximately the common Arrhenius temperature dependence 


$$
D_{S} \propto \exp \left(-\frac{E_{D S}}{R T}\right)
$$

with

$$
E_{D S}=10.43 \mathrm{kcal} / \mathrm{mole}
$$

From eq. 4b, the corresponding dependencies

$$
\tau_{D S} \propto \exp \left(\frac{E_{D S}}{R T}\right)
$$

for the star molecule diffusion correlation time follow. Equations 20-22 hold only for degrees of polymerization in the range $490 \leq N \leq 1200$.

Using the linear and star DHP tracer diffusion results of eqs. $16-22$, the predictions from eq. 8 relating the $M$ - and $T$-dependences of $\tau_{D S}$ to those of $\tau_{D L}$ can be tested. Following the procedure of the last section, the observed Arrhenius temperature dependencies of $\tau_{D S}$ and $\tau_{D L}$ (given by eqs. $17,18,21$, and 22 ) are used in eq. 8 a to determine the coupling parameter, $n_{D S}$, for the three stars DJK5, DJK6, and DWG2:

$$
n_{D S}=0.59
$$

With $n_{D S}$ determined, the variation of $D_{S}$ with molecular weight predicted by eq. $4 \mathrm{~b}$ (valid only within the molecular weight range encompassed by these polymers) is

$$
D_{S} \propto M^{1-2 /\left(1-n_{D}\right)} \propto M^{-3.87}
$$

In Figure 3, this variation ( solid line) is seen to be consistent with the experimental data for DJK5, DJK6, and DWG2.

\section{CONCLUSION}

It is well-established experimentally that the transport coefficients, including zero-shear viscosity, tracer, and self-diffusion coefficient, of entangled star-branched polymers have stronger temperature dependencies than their linear counterparts. Recent work on terminal mechanical relaxation ${ }^{15}$ and dielectric normal mode relaxation $^{16}$ of linear and star polyisoprene demonstrated that this property holds even in a polymer in which the gauche and the trans conformal energies are almost equal. The excess temperature de- pendence of star polymers and their stronger molecular weight dependence are quantitatively related and explainable by the coupling model. Contrarily, expectations based on the reptation model are inconsistent with the linear and branched polymer data.

This work was supported by the Office of Naval Research.

\section{REFERENCES AND NOTES}

1. T. P. Lodge, N. A. Rotstein, and S. Prager, in Advances in Chemical Physics, I. Prigogine, and S. A. Rice, Eds., John Wiley \& Sons, New York, 1990.

2. W. W. Graessley, Acc. Chem. Res. 10, 332 (1979); Adv. Polym. Sci., 47, 67 (1982).

3. M. Doi and S. F. Edwards, The Theory of Polymer Dynamics, Clarendon Press, Oxford, U.K., 1986.

4. J. des Cloizeaux, Macromolecules, 23, 4678 (1990).

5. W. W. Graessley, Macromolecules, 15, 1164 (1982).

6. K. L. Ngai and D. J. Plazek, J. Polym. Sci. Polym. Phys. Ed., 23, 2159 (1985).

7. K. L. Ngai, R. W. Rendell, A. K. Rajagopal, and S. Teitler, Ann. N.Y. Acad. Sci., 484, 150 (1986).

8. G. B. McKenna, K. L. Ngai, and D. J. Plazek, Polymer, 26, 1651 (1985).

9. K. L. Ngai and D. J. Plazek, Rubber Chem. Tech. Rubber Rev., 68, 376 (1995).

10. R. W. Rendell, K. L. Ngai, and G. B. McKenna, Macromolecules, 20, 2250 (1987).

11. K. L. Ngai, A. K. Rajagopal, and T. P. Lodge, J. Polym. Sci., Part B: Polym. Phys., 28, 1367 (1990).

12. R. S. Porter, J. P. Know, and J. F. Johnson, Trans. Soc. Rheol., 12, 409 (1968).

13. J. M. Carella, J. T. Gotro, and W. W. Graessley, Macromolecules, 19, 659 (1986). J. T. Gotro and W. W. Graessley, Macromolecules, 17, 2767 (1984).

14. V. R. Raju, H. Rachapudy, and W. W. Graessley, J. Polym. Sci. Polym. Phys. Ed., 17, 1223 (1979).

15. C. A. Bero and C. M. Roland, Macromolecules, 29, $1562(1996)$.

16. C. A. Bero and C. M. Roland, Macromolecules, 29, 7521 ( 1996 ).

17. K. L. Ngai, Comments Solid State Phys., 9, 127 (1979); K. L. Ngai and C. T. White, Phys. Rev., B20, 2475 ( 1979).

18. K. L. Ngai, S. L. Peng, and K. Y. Tsang, Physica, A191, 523 (1993); K. L. Ngai and R.W. Rendell, J. Non-Cryst. Solids, 131-133, 233 (1991); R. W. Rendell, Phys. Rev. E, 48, R17 (1993); R.W. Rendell, J. Appl. Phys., 75, 7626 (1993); K. L. Ngai and K. Y. Tsang, Phys. Rev. E, 54, R3067 (1996).

19. P. Santangelo, K. L. Ngai, and C. M. Roland, Polymer, to appear.

20. J. E. Mark, Rubber Chem. Tech., 46, 593 (1973). 
21. L. J. Fetters, A. D. Kiss, D. S. Pearson, G. F. Quack, and F. J. Vitus, Macromolecules, 26, 647 (1993).

22. J. T. Gotro and W. W. Graessley, Macromolecules, 17, 2767 (1984).

23. C. M. Roland, Macromolecules, 25, 7031 (1992).

24. J. Klein, D. Fletcher, and L. J. Fetters, Nature (London), 304, 526 (1983); Faraday Symp. Chem. Soc., No. 18, 159 (1983); D. P. Fletcher and J. Klein, J. Polym. Commun. (1985).

25. M. Tirell, Rubber Chem. Technol., 57, 523 (1984) and references therein.

26. Y. Imanishi, K. Adachi, and T. Kotaka, Macromolecules, 19, 7585 (1989); K. Adachi, H. Yoshida, F. Fukui, and T. Kotaka, Macromolecules, 23, 3138 (1990); H. Yoshida, H. Watanabe, K. Adachi, and T. Kotaka, Macromolecules, 24, 2981 (1991); K.
Adachi, T. Nakamoto, and T. Kotaka, Macromolecules, 22, 3111 (1989).

27. K. L. Ngai, R. W. Rendell, and D. J. Plazek, J. Phys. (Paris) Colloq., C8, 6, 555 (1997).

28. R. A. Mendelsohn, W. A. Bowles, and F. L. Fingers, J. Polym. Sci., Part A-2, 8, 105 (1970).

29. R. Bachus and R. Kimmich, Polymer, 24, 3138 (1983); G. Fleischer, Polym. Bull., 9, 152 (1983); 11, 75 (1984); J. Klein and B. J. Briscoe, Proc. $R$. Soc. London A, 365, 53 (1979).

30. N. Nemoto, M. Moriwaki, H. Odani, and K. Michio, Macromolecules, 4, 215 (1971).

31. H. Vogel, Phys. Z., 22, 645 (1921).

32. G. S. Fulcher, Am. Ceram. J., 8, 339 (1925).

33. G. Tamman and W. Z. Hesse, Anorg. Chem. Allg. Chem., 156, 245 (1926). 